Int. J. Dev. Biol. 51: 689-700 (2007)

doi: $10.1387 /$ ijdb.072302ss

Original Article

\title{
Regulation of the mouse $\alpha$ B-crystallin and MKBP/HspB2 promoter activities by shared and gene specific intergenic elements: the importance of context dependency
}

\author{
SHIVALINGAPPA K. SWAMYNATHAN\# and JORAM PIATIGORSKY* \\ Laboratory of Molecular and Developmental Biology, National Eye Institute, NIH, Bethesda, Maryland, USA
}

\begin{abstract}
The closely linked (863 bp), divergently arranged mouse myotonic dystrophy kinase binding protein (Mkbp)/HspB2 and small heat shock protein (shsp)/ $\alpha B$-crystallin genes have different patterns of tissue-specific expression. We showed previously that an intergenic enhancing region (-436/-257 relative to $\alpha B$-crystallin transcription start site) selectively activates the $\alpha B$ crystallin promoter in an orientation-dependent manner (Swamynathan, S.K. and J. Piatigorsky 2002. J. Biol. Chem. 277:49700-6). Here we show that cis-elements $\alpha B E 1(-420 /-396)$ and $\alpha B E 3$ (-320/ -300) functionally interact with glucocorticoid receptor (GR) and Sp1, respectively, both in vitro and in vivo. $\alpha B E 1: G R$ regulates both the HspB2 and $\alpha B$-crystallin promoters, while $\alpha B E 3: S p 1$ selectively regulates the $\alpha B$-crystallin promoter, as judged by mutagenesis and co-transfection tests. Enhancer blocking assays indicate that the $-836 /-622$ fragment can act as a negative regulator in transfection tests, raising the possibility that it contributes to the differential expression of the proximal HspB2 promoter and distal $\alpha B$-crystallin promoter. Finally, experiments utilizing transiently transfected cells and transgenic mice show that two conserved E-box elements (-726/-721 and -702/-697) bind nuclear proteins and differentially regulate the HspB2 and $\alpha B$-crystallin promoters in a tissue-specific manner. Taken together, our results indicate that the linked, differentially expressed $H s p B 2$ and $\alpha B$-crystallin genes have evolved shared and promoterpreferred cis-control elements within the intergenic sequence. The context-dependency of ciselements provides multiple opportunities for evolutionary novelty by small sequence changes.
\end{abstract}

KEY WORDS: crystallin, gene regulation, promoter activity, enhancer, development

\section{Introduction}

Precise tissue-specific gene regulation via trans-acting factors binding to DNA regulatory elements in locus control regions, enhancers and promoters plays a central role in normal development (Wray, 2003). While promoters are adjacent to the transcriptional initiation site of their respective genes, locus control regions and enhancers may be located within introns, at distant positions on the chromosome, or even on separate chromosomes from the genes they regulate (Lee et al., 2006, Lee et al., 2005, Spilianakis and Flavell, 2004, Spilianakis et al., 2005). In addition to the challenge of understanding how distant DNA control elements regulate gene expression or differentially activate multiple genes, there is the problem of understanding how neighboring and potentially shared cis-control elements differentially regulate separate genes linked in a head-to-head arrangement. This is not a trivial issue since more than $10 \%$ of the human genes are closely linked ( 1000 bp), divergently transcribed gene pairs (Adachi and Lieber, 2002, Doerwald, 2004, Labrador and Corces, 2002, Takai and Jones, 2004, Trinklein et al., 2004). Bias towards bidirectional arrangement of genes appears to be the result of recent genome reorganizations and a unique feature of mammalian genomes (Koyanagi et al., 2005). It has been suggested that the origin and evolution of bidirectional arrangement of genes may have its roots in the selective pressure imposed by the shared cis elements in the intergenic region on the expression patterns of the flanking genes (Adachi and Lieber, 2002, Koyanagi et al., 2005, Takai and Jones, 2004, Trinklein et al., 2004).

The small heat shock protein (shsp)/ $\alpha$ B-crystallin and the related myotonic dystrophyprotein kinase binding protein (Mkbp)/

Abbreviations used in this paper: DPE, downstream promoter element; GR, glucocorticoid receptor; mkbp, myotonic dystrophy kinase binding protein; NRE, negative regulatory element; shsp, small heat shock protein.

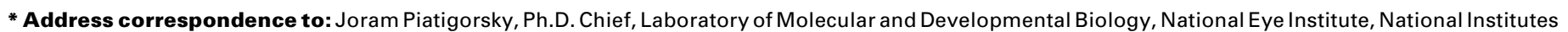
of Health, 7 Memorial Drive, Room 101, Bethesda, MD 20892, USA. Fax: +1-301-402-0781. e-mail: joramp@nei.nih.gov
} 
A
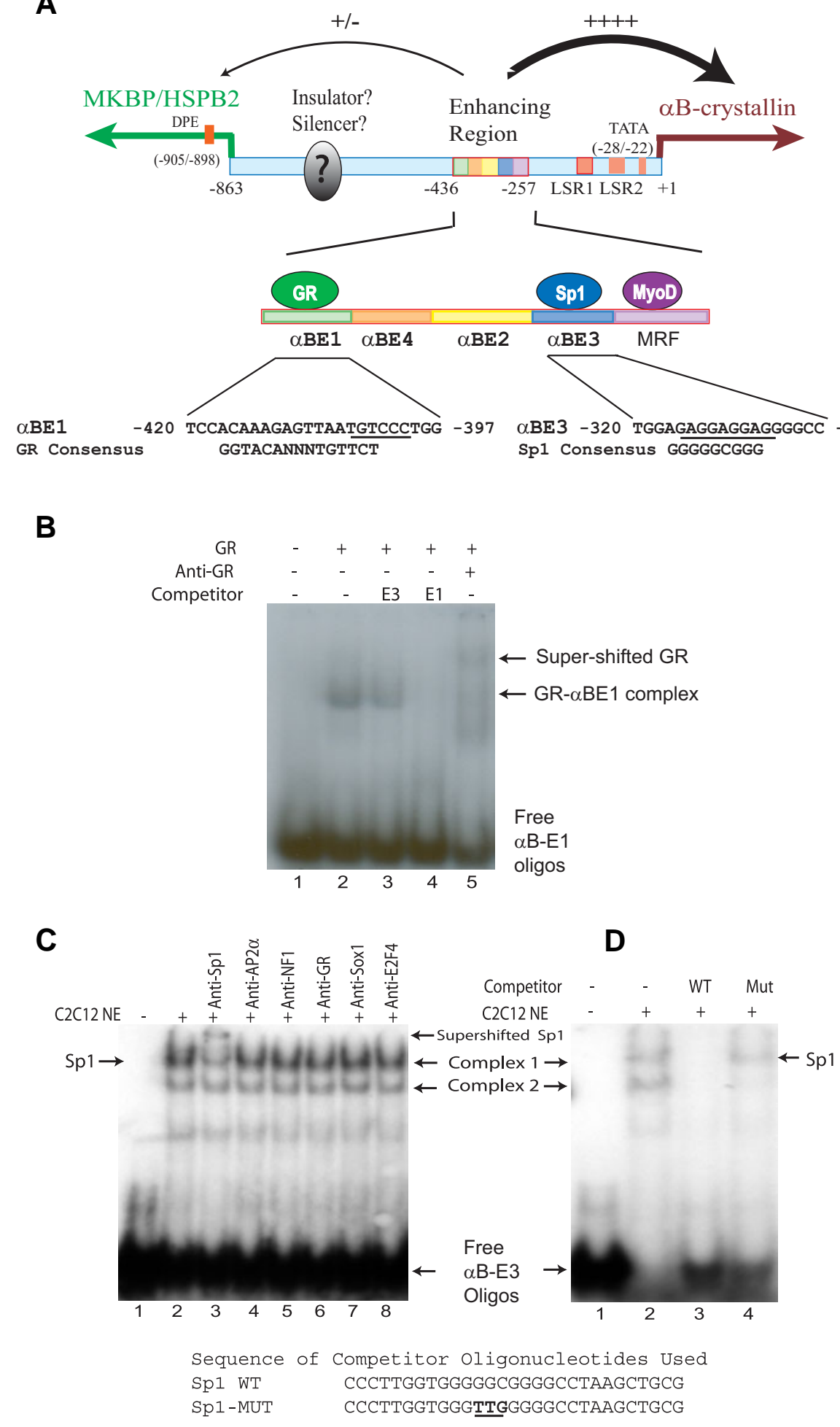

HspB2genes provide a useful model of how shared intergenic sequences regulate the expression of divergently linked genes. For simplicity we refer to these genes as $\alpha B$ crystallin and HspB2. The $\alpha \mathrm{B}$-crystallin protein is one of several lens crystallins - the abundant, water-soluble, cytoplasmic proteins that contribute to the transparent and refractive properties of the ocular lens - found in all vertebrates (de Jong et al., 1989, Horwitz, 2003, Wistow and Piatigorsky, 1988). In mammals, at least ten members of the shsp family are expressed either in a constitutive or stress-inducible manner in different tissues (de Jong et al., 1998, Franck et al., 2004, Kappe et al., 2003). HspB2 is a novel member of the shsp family that binds and activates myotonic dystrophy protein kinase (Iwaki et al., 1997, Suzuki et al., 1998). In mice, $\alpha B$-crystallin and HspB2 are arranged divergently with their transcription start sites separated by 863 bp (Iwaki et al., 1997, Suzuki et al., 1998) (Fig. 1A).

The expression patterns of the $\alpha B$-crystallin and $H s p B 2$ genes differ despite the presence of a small intergenic sequence containing gene regulatory elements that can be potentially shared. The $\alpha B$-crystallin gene is inducible by physiological stress (Klemenz et al., 1991) and is expressed abundantly in the lens and heart, moderately in the diaphragm, intestine and skeletal muscle and at low levels in kidney, spleen, liver, brain and retina (Bhat and Nagineni, 1989, Dubin etal., 1989, Haynes et al., 1996, Xi et al., 2003). By contrast, the HspB2gene is neither stress inducible nor expressed in the lens and is expressed at low levels in the

Fig.1. Interaction of GR and Sp1 with elements $\alpha B E 1$ and $\alpha B E 3$, respectively. (A) Schematic showing the known cis elements in the intergenic region of the mouse $\alpha \mathrm{B}$-crystallin and HspB2 genes. The -436/-257 intergenic enhancing region is expanded to show the organization of different cis elements. LSR1 and LSR2, lens specificity region 1 and 2, respectively. DPE, downstream promoter element. Sequence of elements $\alpha B E 1$ and $\alpha B E 3$, along with the consensus recognition sites of $G R$ and $S p 1$ respectively, are shown. The glucocorticoid response element half site within the element $\alpha B E 1$ and

the GAG trinucleotide repeats known to interact with transcription factors GR and Sp 1 respectively are underlined. (B) Interaction of purified GR with $\alpha B E 1$. Double-stranded radiolabeled $\alpha B E 1$ oligonucleotides were incubated with (lanes 2-5) or without (lane1) purified GR. Specific double-stranded oligonucleotide competitors were included as indicated in lanes 3 and 4 . The free $\alpha B E 1$ oligonucleotides, mobility shifted $\alpha B E 1+G R$ complex (lanes 2 and 3) and super-shifted $\alpha B E 1+G R+A n t i-G R$ complex (lane 5) are indicated by arrows. (C) Interaction of Sp1 with labeled element $\alpha B E 3$. Doublestranded labeled $\alpha B E 3$ oligonucleotides were incubated with or without C2C12 nuclear extracts in the presence of different antibodies as shown. The slow-moving complex 1, which was super-shifted in the presence of anti-Sp1 antibody (lane 3), is indicated. (D) Double-stranded labeled $\alpha B E 3$ oligonucleotides incubated with C2C12 nuclear extracts in the presence of 100 molar excess of double-stranded competitors, Sp1 recognition site containing wild type oligonucleotides (WT) or mutant oligonucleotides not capable of binding Sp1 (Mut). 
skeletal muscle, heart and intestine (Iwaki et al., 1997, Suzuki et al., 1998, Swamynathan and Piatigorsky, 2002). A number of DNA regulatory elements have been identified for the mouse $\alpha B$ crystallingene. These include two ciselements (LSR1 and LSR2) conferring lens-specificity that interact with Pax6, Maf and RXR in the proximal promoter (Cvekl et al., 2004, Gopal-Srivastava et al., 1996, Gopal-Srivastava et al., 1998, Gopal-Srivastava et al., 1995, Gopal-Srivastava and Piatigorsky, 1994, Haynes et al., 1997, Yang etal., 2004) and an upstream enhancing region (-436/ -258) containing at least 5 distinct cis elements in the intergenic sequence that increases $\alpha B$-crystallin promoter activity in the lens and other tissues (especially heart and skeletal muscle) (GopalSrivastava et al., 1995, Gopal-Srivastava and Piatigorsky, 1993, Swamynathan and Piatigorsky, 2002). No comparable studies have been performed delineating the cis-elements regulating HspB2 gene expression. Phylogenetic footprint analysis by sequence alignment indicated that LSR1, LSR2 and the 5 cisregulatory elements within the enhancer are conserved in mammals (Doerwald, 2004) (Fig. 1A).

We showed earlier that the intergenic enhancing region preferentially augments $\alpha \mathrm{B}$-crystallin promoter activity and is orientation-dependent in its natural context (Swamynathan and Piatigorsky, 2002), both traits consistent with the differences in the endogenous expression patterns of the $\alpha B$-crystallin and $H s p B 2$ genes. In addition, we suggested the existence of an insulator between the enhancing region and HspB2 promoter on the basis of sequence analysis and previously published transgenic mouse results (Swamynathan and Piatigorsky, 2002). In the present investigation we identify one cis-control element ( $\alpha \mathrm{BE} 3$, a Sp1-responsive site) within the enhancing region and two upstream $E$-boxes in the intergenic region that favor $\alpha \mathrm{B}$-crystallin promoter activity, another ciscontrol element ( $\alpha B E 1$, a glucocorticoid responsive site) in the enhancing region that equally affects the activity of the two promoters and a sequence between the enhancing region and $\mathrm{HspB} 2$ promoter that acts as a negative regulatory element when tested in transfected cells in culture. These results provide new molecular insights suggesting that the differential use of shared cis-control elements contributes to the differences in the expression of the divergently transcribed $\alpha B$-crystallin and $H s p B 2$ genes in the mouse.

\section{Results}

\section{Candidate transcription factors for interaction with $\alpha B E 1$ and $\alpha B E 3$ within the enhancing region}

Although 5 regulatory motifs (5‘ $\alpha \mathrm{BE} 1, \alpha \mathrm{BE} 4, \alpha \mathrm{BE} 2$, $\alpha B E 3$ and MRF $3^{\prime}$ ) have been delineated in the $\alpha B$ crystallin enhancing region by DNase footprinting and functional studies (Gopal-Srivastava et al., 1995, GopalSrivastava et al., 2000, Gopal-Srivastava and Piatigorsky, 1993, Haynes etal., 1995), few of the cognate transcription factors are known. We thus screened the TRANSFAC database of transcription factor recognition sites (http:// www.cbil.upenn.edu/tess) (Schug, 2003) and identified the glucocorticoid receptor (GR) and Sp1 as candidate transcription factors for binding to sequences within $\alpha B E 1$ and $\alpha B E 3$, respectively. Even though glucocorticoid re- sponse elements (GRE) typically consist of a palindrome that can interact with a hormone bound GR dimer, GRE half sites also are effective in several promoters (Schoneveld et al., 2004). The nucleotide sequence within $\alpha B E 1$ consists of a GRE half site, to which a GR monomer may bind (Segard-Maurel et al., 1996) (Fig. 1A). Similarly, even though the hexanucleotide core GGGCGG is considered critical in the consensus Sp1 recognition site 5'-(G/ T)GGGCGG(G/A)(G/A)(C/T)-3', variations such as three repeats of the trinucleotide $\mathrm{GAG}$ present within $\alpha \mathrm{BE} 3$ can bind Sp1 with high affinity (Marco et al., 2003) (Fig. 1A). Based on these considerations, we pursued investigation of GR and Sp1 as candidates for interacting with elements $\alpha B E 1$ and $\alpha B E 3$, respectively.

\section{Interaction of GR and Sp1 with $\alpha B E 1$ and $\alpha B E 3$}

Interaction of GR with $\alpha \mathrm{BE} 1$ was tested directly by incubating partially purified GR expressed in insect cells with radioactively labeled $\alpha B E 1$ oligonucleotides. GR formed a nucleotide sequence-specific complex with $\alpha \mathrm{BE} 1$, which could be competed by non-labeled double-stranded $\alpha B E 1$ but not $\alpha B E 3$ oligonucleotide (Fig. 1B). Incubation of anti-GR antibody in the reaction mix resulted in a super-shift of the GR complex, consistent with GR interacting with the enhancing element $\alpha B E 1$ (Fig. 1B).

In electrophoretic mobility shift assays using labeled oligonucleotide $\alpha \mathrm{BE} 3$ incubated with nuclear extracts from $\mathrm{C} 2 \mathrm{C} 12$ muscle cells, 2 distinct complexes were observed (Fig. 1C). AntiSp1 antibody super shifted complex 1, consistent with Sp1 being a component of this complex. Treatment with other available

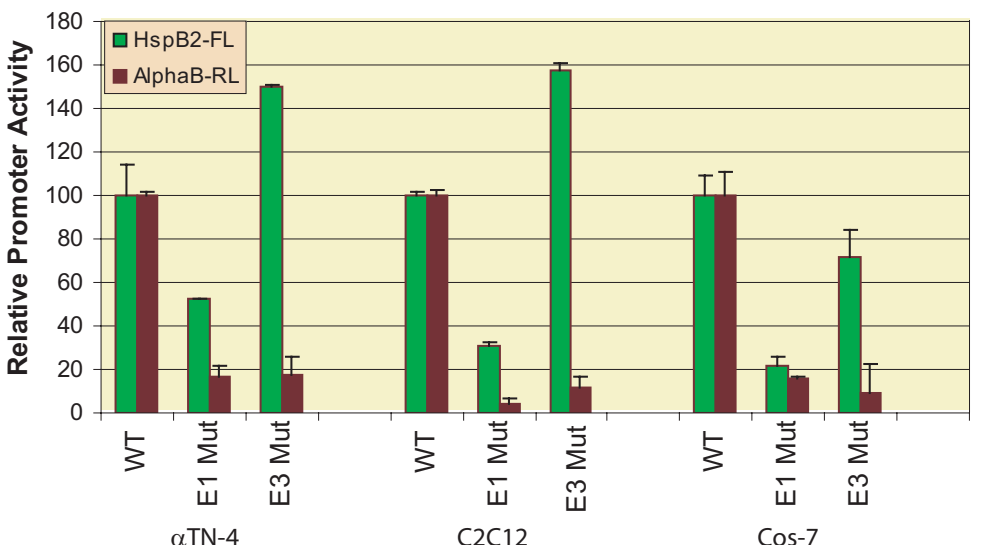

Wild Type $\alpha$ BE1:-426 TAGGACTCCACAAAGAGTTAATGTCCCTGGGG Mutant $\alpha$ BE1: $\quad$-426 TAGGACAGTCAGTCCTCACTCAACTTGACTGG

Wild Type $\alpha$ BE $3:-326$ CGAGACTGGAGAGGAGGAGGGGCCACCAG Mutant $\alpha$ BE3: $\quad-326$ CGAGACTCATATCTGACTACCATGCACCAG

Fig. 2. Transient transfection assays showing the effect of mutations in elements $\alpha B E 1$ or $\alpha B E 3$ on $\alpha B$-crystallin and HspB2 promoter activities. Wild type, $\alpha B E 1$ mutant or $\alpha B E 3$ mutant $p F L-H s p B 2 \alpha B-R L$ plasmids were transfected into $\alpha \mathrm{TN}-4, \mathrm{C} 2 \mathrm{C} 12$ or Cos-7 cells. $\alpha B$-crystallin and HspB2 promoter activities measured using the $\alpha B E 1$ mutant or $\alpha B E 3$ mutant plasmids are shown relative to that obtained using the wild type plasmid. Nucleotide sequence of wild type and mutant elements $\alpha B E 1$ and $\alpha B E 3$ is shown below. Note that the endogenous expression level of $\alpha B$-crystallin is about 30-100 fold higher than that of HspB2 in different tissues. Here, each mutant promoter activity is shown relative to the respective wild type promoter activity, which is set to 100 . 

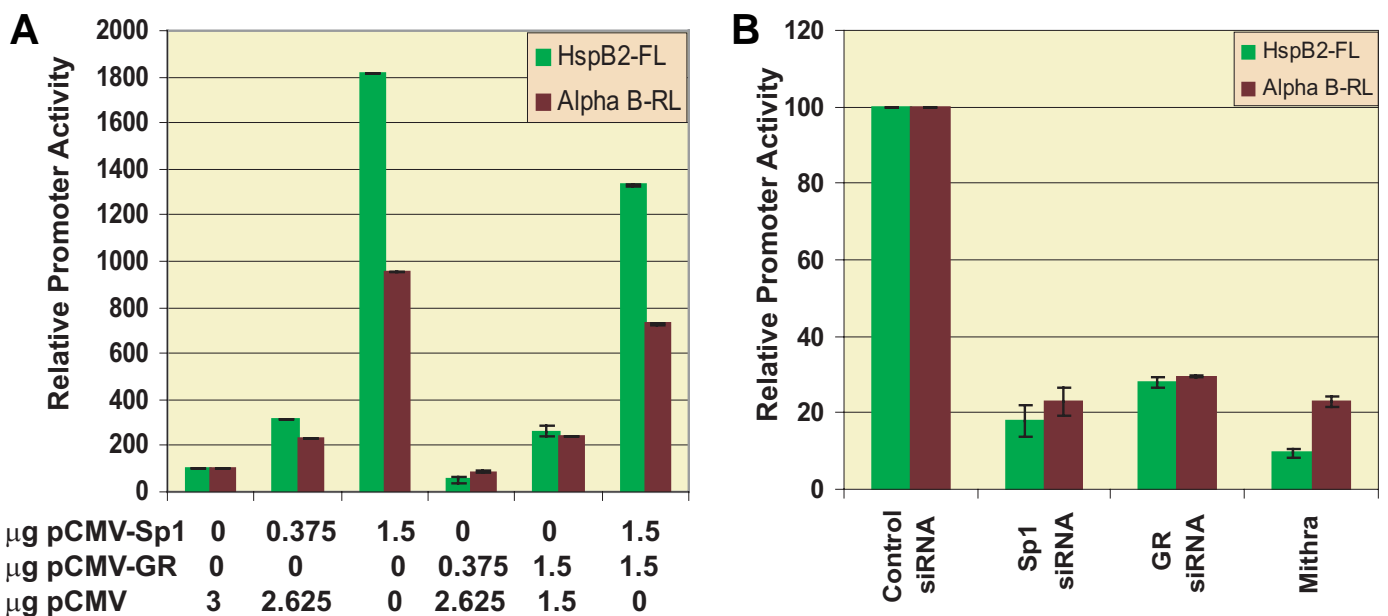

Fig. 3. Transient co-transfection assays showing the effect of modulating the intracellular levels of Sp1 or GR on $\alpha B$-crystallin and HspB2 promoter activities. (A) Effect of over-expression of Sp1 or GR. $\alpha B$-crystallin and HspB2 promoter activities obtained upon co-transfection with plasmids $p F L-H s p B 2 \alpha B-R L$ and increasing amounts of $\mathrm{pCMV}$-Sp1 or pCMV-GR in Cos-7 cells are shown relative to those obtained in co-transfections with empty vector $p C M V$. (B) Effect of suppression of $\mathrm{Sp} 1$ or GR. $\alpha B$-crystallin and HspB2 promoter activities obtained upon co-transfection with plasmid pFL-HspB2 $\alpha B$-RL and specific siRNAs to Sp1 or GR in Cos-7 cells are shown relative to those obtained in co-transfections with control siRNAs. Effect of treatment with mithramycin-A (mithra), a specific inhibitor of Sp1, on $\alpha B$-crystallin and HspB2 promoter activities also is shown. Note that the endogenous expression level of $\alpha B$-crystallin is about 30-100 fold higher than that of HspB2 in different tissues. Here, each promoter activity is shown relative to the respective basal promoter activity, which is set to 100.

antibodies to different transcription factors (AP2- $\alpha$, NF1, GR, Sox1 and E2F4) did not affect the formation of complex 1 (Fig. 1C). In competition experiments, inclusion of 100 molar excess of nonlabeled Sp1 consensus double-stranded oligonucleotides in the reaction mix abolished the formation of complexes 1 and 2 (Fig. 1D). When similar amounts of oligonucleotides mutated in the Sp1 binding site were used for competition, only complex 2 was abolished (Fig. 1D). From these results, we conclude that the complex 1 includes Sp1, while complex 2 does not.

\section{Mutational analysis of $\alpha B E 1$ and $\alpha B E 3$}

In order to test the functional roles of $\alpha \mathrm{BE} 1$ and $\alpha \mathrm{BE} 3$ on the $\alpha \mathrm{B}$ crystallin and $\mathrm{HspB} 2$ promoters, we introduced specific mutations in elements $\alpha B E 1$ or $\alpha B E 3$ by recombinant PCR-mediated ligation and cloned the mutated fragments in between the firefly and Renilla luciferase reporter genes in the bidirectional dual reporter vector (Swamynathan and Piatigorsky, 2002). In this vector, the expression of the divergently arranged firefly and Renillaluciferase reporter genes is controlled by the HspB2 and $\alpha \mathrm{B}$-crystallin pro-

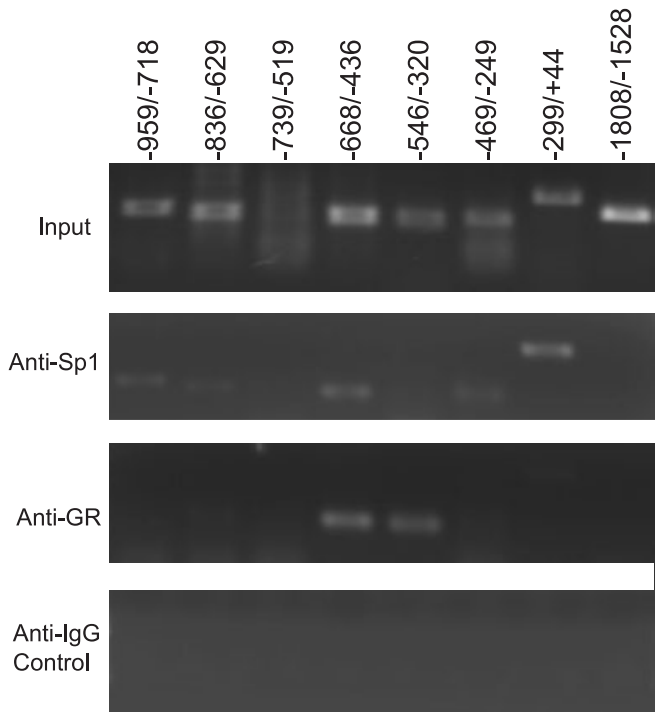

moters respectively, mimicking the endogenous organization of the HspB2and $\alpha B$-crystallingenes. Transient transfection analysis using $\alpha \mathrm{TN}-4, \mathrm{C} 2 \mathrm{C} 12$ or Cos-7 cells showed that the mutation in $\alpha B E 1$ reduced the $\alpha B$-crystallin and $\mathrm{HspB} 2$ promoter activities by 6 to 10 -fold and 2 to 5 -fold, respectively (Fig. 2). Upon mutagenesis of $\alpha \mathrm{BE} 3, \alpha \mathrm{B}$-crystallin promoter activity was reduced by 6 to 10 fold, while HspB2 promoter activity was marginally increased in $\alpha \mathrm{TN}-4$ and C2C12 cells (by 1.5- and 1.58-fold, respectively) and marginally decreased in Cos-7 cells (to $72 \%$ of the WT) (Fig. 2). Therefore, $\alpha B E 1$ activates the flanking promoters in a bidirectional manner, while $\alpha B E 3$ activates the $\alpha B$-crystallin promoter in a unidirectional manner with a moderate, variable and cell type dependent influence on HspB2 promoter.

\section{Effects of modulating the intracellular levels of Sp1 and GR on $\alpha \mathrm{B}-$ crystallin and HspB2 promoter activities}

The effect of increasing the intracellular levels of Sp1 and GR on $\alpha B$-crystallin and HspB2 promoter activities was studied by cotransfection experiments with plasmids expressing Sp1 or GR. Cos-7 cells were used for these experiments because they support a relatively lower level of $\alpha \mathrm{B}$-crystallin promoter activity than the transfected $\alpha \mathrm{TN}-4$ and $\mathrm{C} 2 \mathrm{C} 12$ cells, thus obviating a high background difficulty. $\alpha \mathrm{B}$-crystallin and $\mathrm{HspB} 2$ promoter activities were upregulated 10 and 18-fold respectively, by pCMV-Sp1 and 3 and 2.5-fold respectively, by pCMV-GR (Fig. 3A). There was no synergistic upregulation when pCMV-Sp1 and PCMV-GR were used

Fig. 4. Identification of in vivo association of Sp1 and GR with the intergenic fragment by chromatin immunoprecipitation (ChIP). Crosslinked chromatin isolated from C2C12 cells was immunoprecipitated with anti-Sp1, anti-GR or negative control anti-lgG antibodies. After cross links were removed and the associated chromosomal DNA fragments purified, PCR was performed with specific primer pairs. PCR products resulting from different starting materials, (i) input DNA, (ii) anti-Sp1 antibody immunoprecipitated DNA, (iii) anti-GR antibody immunoprecipitated DNA and (iv) negative control anti-lgG immunoprecipitated DNA used as target are shown. Nucleotide position of each amplified fragment is shown on top. 
together in co-transfection experiments (Fig. 3A). The effect of decreasing the intracellular levels of Sp1 and GR on $\alpha B$-crystallin and $\mathrm{HspB} 2$ promoter activities was studied using specific siRNAs. Compared to control siRNAs, co-transfection of Sp1- or GRspecific siRNAs resulted in approximately 5-fold reductions in $\alpha B$ crystallin and HspB2 promoter activities in Cos-7 cells (Fig. 3B). In other tests, when transfected cells were treated with mithramycin$A$, a specific inhibitor of transcription factor Sp1 (Christensen et al., 2004), $\alpha B$-crystallin and HspB2 promoter activities decreased by 5 and 10-fold, respectively (Fig. 3B).

In vivo interactions of Sp1 and GR with the intergenic region

Mutations in the Sp1 binding element $\alpha B E 3$ selectively lowered $\alpha \mathrm{B}$-crystallin promoter activity (Fig. 2). However, co-transfection experiments demonstrated that the activities of both the $\alpha B$ crystallin and HspB2 promoters are elevated in response to Sp1 (Fig. 3). A likely explanation for this apparent discrepancy is that there are additional Sp1 binding sites within the intergenic region. Consistent with the idea that Sp1 influences the activity of $\alpha \mathrm{B}$ crystallin and HspB2 promoters at multiple binding sites, several potential Sp1-binding sites were identified within the intergenic region (-860/-853, -820/-800, -748/-738, -399/-388, -250/-240 and $-21 /-10)$ by the transcription element search software (URL: http:/ /www.cbil.upenn.edu/tess) (Schug, 2003). Evidence that functional Sp1 binding sites occur at these sites was obtained by scanning the intergenic region by ChIP analysis. Sp1 was associated with the following fragments along the intergenic region: -959/ $-718,-836 /-629,-668 /-436,-469 /-249$ and $-299 /+44$. By contrast, GR was associated only with the -668/-436 and -546/-320 fragments (Fig. 4). The precise location of individual Sp1- or GRbinding cis-elements within these fragments remains to be confirmed by other methods. Association of Sp1 with numerous sites explains why both the promoters are responsive to Sp1 even though the Sp1- binding site $\alpha B E 3$ selectively activates the $\alpha B$ crystallin promoter.

Features of the nucleotide sequence between the $\alpha B$-crystallin enhancing region and the HspB2 promoter

A discrete AT- rich stretch immediately upstream of the enhanc- ing region (-636/-436 fragment, $42 \%$ GC content) is followed by a GC-rich stretch proximal to the HspB2 promoter (-836/-636, $62 \%$ GC content) (Fig. 5A). Within the GC-rich -836/-636 fragment, there are 14 repeats of CCCTC (or one nucleotide variants of this motif) that show extensive similarities with binding sites for CTCF (Chung et al., 1997, Farrell et al., 2002, Filippova et al., 1996, Ohlsson et al., 2001, Vostrov and Quitschke, 1997), the insulatorbinding zinc finger protein (Bell and Felsenfeld, 1999, Bell et al., 1999, Ohlsson et al., 2001) (Fig. 5B). Insulators are positiondependent enhancer blockers that restrict the effects of an enhancer to a specific promoter in the vicinity of multiple promoters. Interaction with the zinc-finger protein CTCF is both necessary and sufficient for insulators to work (Bell and Felsenfeld, 1999, Bell et al., 1999, Bell etal., 2001). We thus tested the $-836 /-519$ sequence for the presence of insulator activity by an enhancer blocking assay. An insulator located between the $\alpha \mathrm{B}$-crystallin enhancing region and the $\mathrm{HspB} 2$ promoter would contribute to the selective influence of the enhancing region on the $\alpha \mathrm{B}$-crystallin promoter.

\section{Enhancer blocking assays reveal a potential NRE in the -836/ -622 sequence}

Enhancer blocking assays using stable transfection of linear fragments of DNA integrated into the genome are useful for identifying insulator elements (Chung et al., 1997, Chung et al., 1993, Recillas-Targa et al., 1999). In the present experiments we cloned overlapping fragments from the -836/-519 intergenic sequence $3^{\prime}$ or $5^{\prime}$ to the globin enhancer in a plasmid (pNI CD) containing the neomycin resistance gene driven by the globin promoter (Fig. 5C) and tested for the number of colonies resistant to G418.

The -836/-519 fragment inserted between the globin enhancer and promoter blocked the effect of the globin enhancer on promoter activity in an orientation-independent manner (Fig. 5C). The 3-4 fold reduction due to the -836/-519 fragment was comparable to the 4-5 fold reduction achieved with the known globin FII insulator in pNI CD (Chung et al., 1997), which was used as a positive control in these tests. When smaller overlapping sequences were tested, only the $-836 /-622$ fragment was an effective insulator. Neither the $-785 /-572$ nor the $-739 /-519$ fragment
Fig. 5. Identification of a negative regulatory element (NRE) in between the $\alpha B$-crystallin enhancing region and the HspB2 promoter. Enhancer blocking assays identify an NRE in -836/-622 bp region. (A) A schematic showing the different fragments tested. pNI in which the globin promoter and enhancer drive the expression of the neomycin resistance gene is the base vector. Filled in triangle represents the chicken $\beta$ globin core insulator; empty triangles represent different fragments tested from the intergenic region, as shown. (B) The average percentage of G418resistant colonies obtained with different constructs relative to that obtained with pNI.

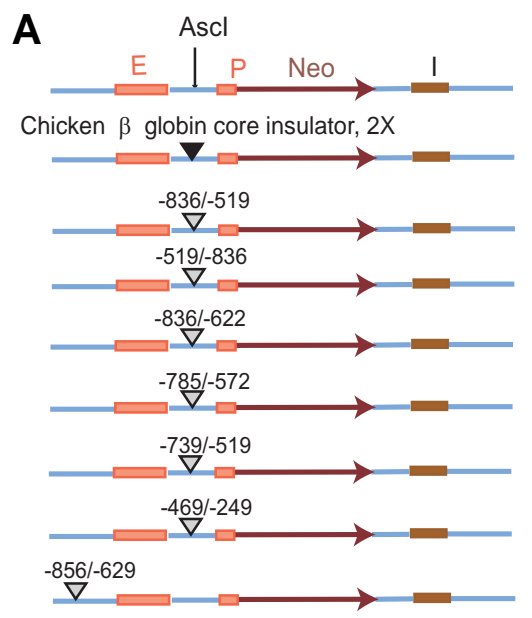

B

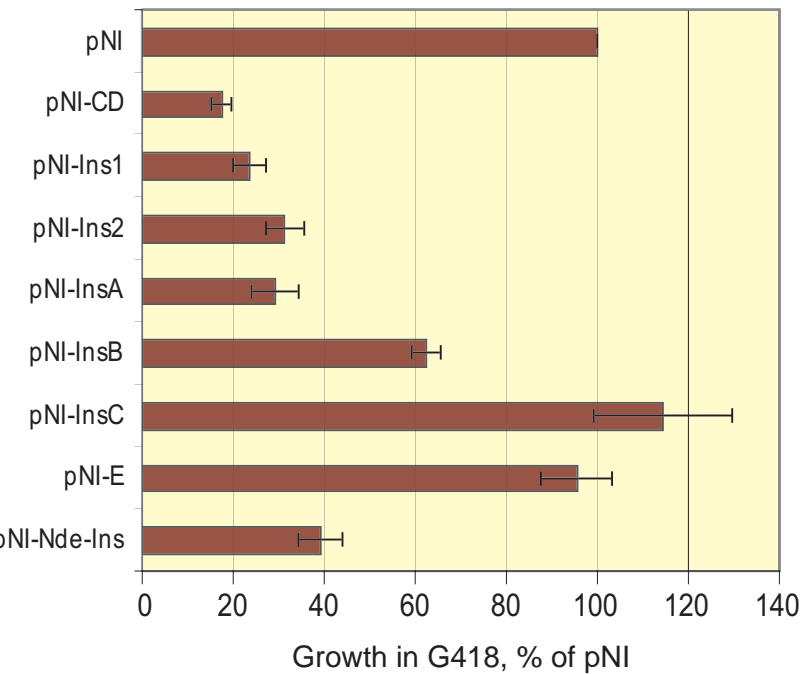


showed insulator activity, consistent with the core insulator activity residing within the $-836 /-739$ fragment. The $-469 /-249$ enhancing region fragment, used as a negative control for insulator function, did not affect globin promoter activity. Surprisingly however, the $-856 /-629$ fragment cloned 5 ' to the enhancerpromoter-neomycin resistancegene cassette (pNI-Nde-856/-629) also reduced globin promoter activity (Fig. 5). Since insulators are position-dependent while negative regulatory elements (NREs) are not, these results suggest that the -836/-739 fragment harbors a NRE as well as, if not instead of an insulator.

\section{Interaction of nuclear factors with the NRE}

In order to identify the nucleotides within the $-836 /-628$ fragment interacting with nuclear factors, we performed DNase I
A

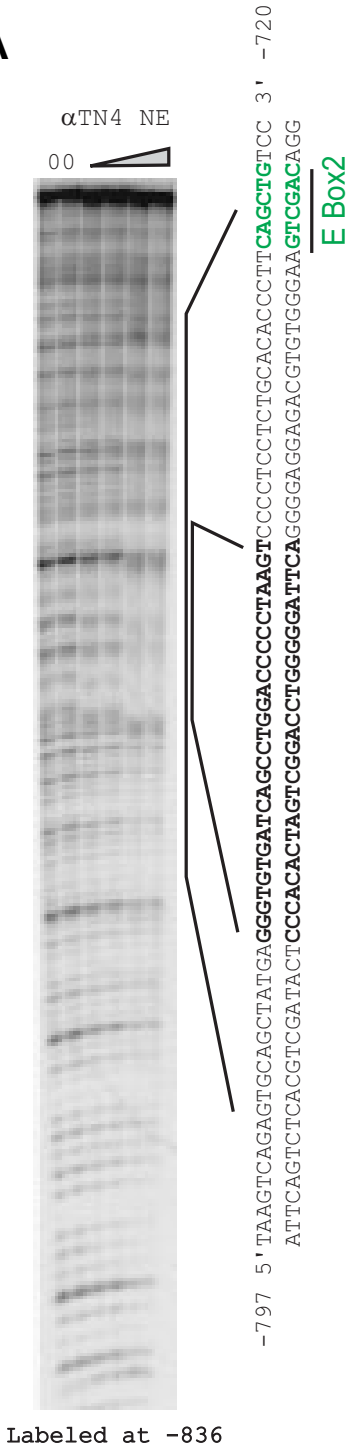

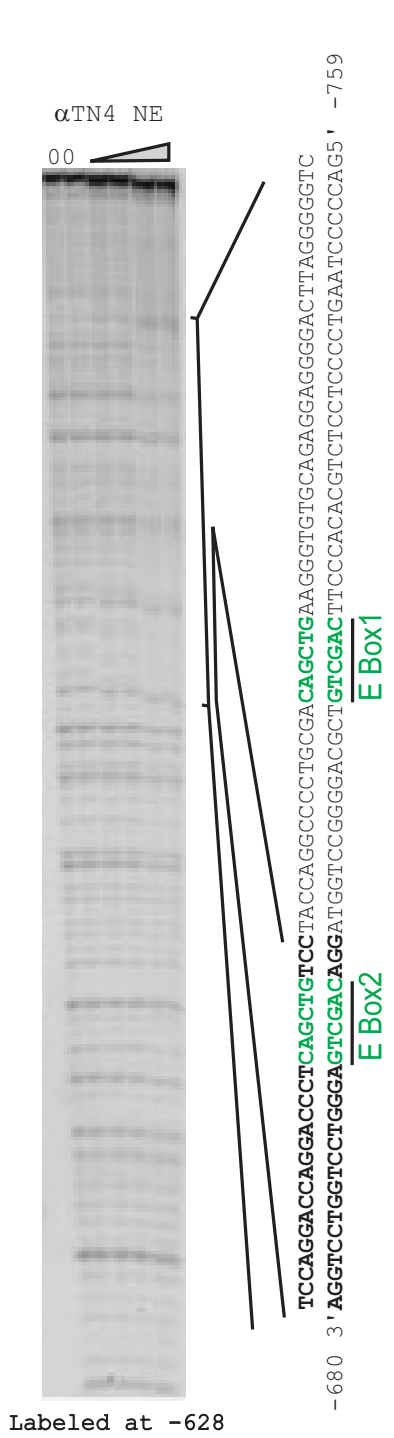

B



C

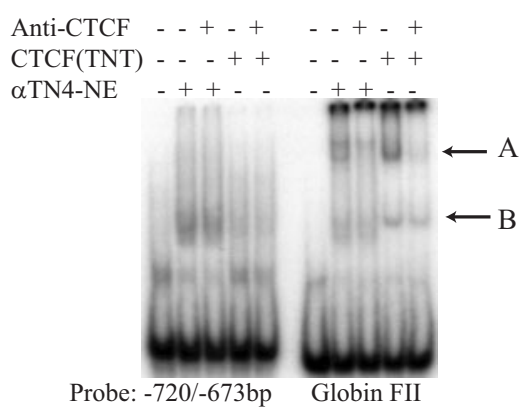

D

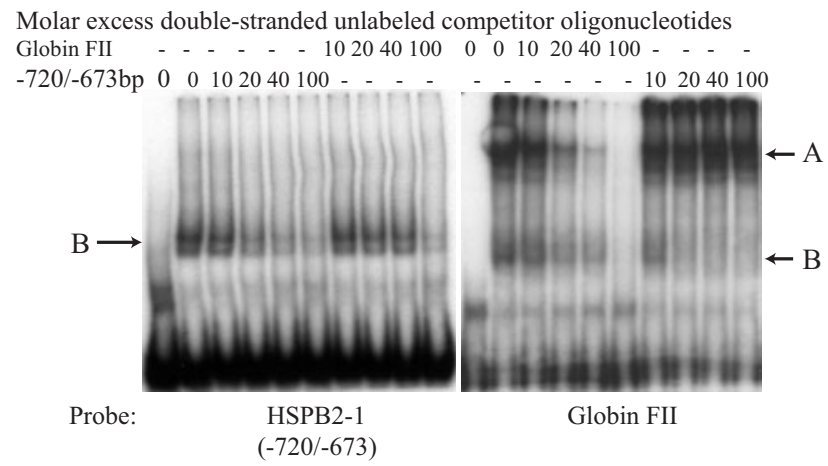

Fig. 6. Interaction of nuclear proteins with the -836/-628 fragment. (A) DNase I footprint analysis of the -836/-628 fragment with nuclear extracts from lens epithelial $\alpha T N-4$ cells. Results obtained with the DNA fragment labeled at the -836 end (left panel) and at -628 end (right panel) are shown. Sequence of protected nucleotides deduced from a sequencing reaction run in parallel is shown by the side. The sequence of the two conserved Ebox elements is indicated. (B) The insulator protein CTCF does not interact with different oligonucleotides from the -836/-629 fragment. Electrophoretic mobility shift assays with partially purified CTCF expressed as 6 X histidine fusion protein in E. coli incubated with different labeled oligonucleotides (see Materials and Methods for their sequence). While the positive control globin Fll oligonucleotides were efficiently bound by CTCF, none of the tested oligonucleotides from the -836/-629 region showed similar binding. (C) Electrophoretic mobility shift assays with $\alpha$ TN-4 nuclear extracts or CTCF produced in vitro by coupled transcription and translation reactions using rabbit reticulocyte lysate and anti-CTCF antibody as shown. Note that the formation of complex A with globin Fll oligonucleotides is abolished in the presence of anti-CTCF antibody, indicating that CTCF is responsible for this complex. Comparable complex A was not formed by the -720/-673 oligonucleotide. (D) Competition with increasing amounts of unlabeled double-stranded oligonucleotides as shown using either labeled-720/-673 oligonucleotides (left panel) or labeled globin Fll oligonucleotides (right panel). Note that the complex B in both panels is competed by both globin Fll and-720/-673 oligonucleotides, while the complex A due to CTCF is competed only by globin Fll oligonucleotides and not by -720/-673 oligonucleotides. 
footprint analysis. Extended regions of protection after DNase I digestion were present between sequences -797 and -680 , within which there were shorter stretches of stronger protection (Fig.6A). The DNase I-protected regions corresponded to the most conserved nucleotides in this region including the two E-box elements identified by sequence comparison across different species (Doerwald, 2004), consistent with these sequences having an important role to play in the regulation of the flanking genes. Furthermore, the protected sequences correlated with the putative CTCF-binding sites, raising the possibility that the protein factor responsible for protection from DNase I may be CTCF.

In order to test whether CTCF interacts with the $-797 /-680$ fragment we performed electrophoretic mobility shift assays (EMSAs) with histidine-tagged CTCF protein expressed in $E$. coli and with various end-labeled oligonucleotides from the $-845 /-629$ fragment. While the positive control oligonucleotides comprising the globinFII insulator bound CTCF, none of the oligonucleotides from the $-845 /-629$ intergenic region did (Fig. 6B). EMSAs performed with $\alpha \mathrm{TN} 4$ mouse lens epithelial cell nuclear extracts or with CTCF produced in vitro by coupled transcription and translation reactions showed that the control globin FIl insulator bound CTCF, while the -720/-673 intergenic fragment did not (Fig. 6C). We obtained similar results with other oligonucleotides from the -845/-629 fragment (data not shown). In competition experiments, complex B was abolished with increasing molar excess of the unlabeled $-720 /-673$ fragment or of the globin FII insulator (Fig. 6D). However, the formation of complex A (CTCF) was competed only by the non-labeled globin FII insulator but not by the -720/-673 oligonucleotide. Based on these results, we conclude that CTCF has little if any, affinity for the -720/-673 oligonucleotide (Fig. 6D). Taken together, these results are consistent with the $-836 /-629$ intergenic fragment being a negative regulatory element (NRE) rather than a CTCF-binding insulator.

\section{Regulatory activity of two E-box elements}

Our DNase I footprint showed that a pair of well conserved Ebox elements (-726/-721 and -702/-697) (Doerwald, 2004) contained within the putative NRE bind nuclear proteins (see Fig. 6A). Clusters of E-box elements are known to be associated with negative (Naghavi et al., 2001, Neuman et al., 1993, Tesmer et al., 1993, Yan et al., 2001, Yoshida et al., 2001) and/or positive regulatory activity (Calomme et al., 2002, Kraner et al., 1998, Nguyen et al., 2003). In order to test if any regulatory activity is associated with these putative E-box binding sites, we introduced point mutations in either E-box1 (-726/-721) or E-box2 (-702/697 ), or both (Fig. 7A) and then performed functional tests using transient transfection assays and transgenic mice. Mutations in either of the $\mathrm{E}$-box elements reduced the $\alpha \mathrm{B}$-crystallin promoter activity near basal levels without affecting the low level of HspB2 promoter activity in transfected C2C12 cells (Fig. 7B). Unexpectedly, the $\alpha \mathrm{B}$-crystallin promoter activity was partially regained when both E-box elements were mutated (Fig. 7B). Similar results were obtained in $\alpha \mathrm{TN}-4$ and Cos-7 cells (results not shown). In transgenic mice, both $\alpha \mathrm{B}$-crystallin and HspB2 promoter activities were reduced in lens, heart, skeletal muscle and lung, although to differing extents, as a result of mutations in either or both of the E-box elements (Fig. 7C). In liver, however, the mutant transgenes were more active than their wild type counterpart and there were small and variable effects in kidney and spleen (Fig.
7C). In spite of the proximity of the E-box elements to the HspB2 promoter, the $\alpha \mathrm{B}$-crystallin promoter activity was proportionally more affected than the HspB2 promoter in these mutants (Fig. 5C). Taken together the data suggest that E-box1 (-726/-721) and E-box2 (702/-697) can have variable positive or negative, tissuespecific effects on $\alpha \mathrm{B}$-crystallin and HspB2 promoter activities.

\section{Discussion}

We showed previously that the intergenic enhancing region between the $\alpha B$-crystallin and $H s p B 2$ genes preferentially influences the $\alpha \mathrm{B}$-crystallin promoter and that the $\alpha \mathrm{B}$-crystallin promoter is $30-100$ fold more active than the HspB2 promoter in different tissues (Swamynathan and Piatigorsky, 2002). The previous data also showed, unexpectedly, that the intergenic enhancing region is orientation-dependent within its normal context, resulting in strong preferential activation of the $\alpha \mathrm{B}$-crystallin promoter over the HspB2 promoter (Swamynathan and Piatigorsky, 2002). Here we report on four different intergenic control elements that contribute to the regulation of the linked, divergently transcribed mouse $\alpha B$-crystallin and $H s p B 2$ genes. Among the cis- elements within the enhancing region, $\alpha \mathrm{BE} 1$ activates both the $\alpha \mathrm{B}$-crystallin and HspB2 promoters equally, while $\alpha \mathrm{BE} 3$ selectively activates the $\alpha B$-crystallin promoter. Two conserved E-box elements located upstream of the enhancing region affect both the promoters, albeit the $\alpha B$-crystallin promoter preferentially, in a tissue-specific fashion. Finally, the fragment located between the E-box elements and the HspB2 promoter has negative regulatory potential when tested with foreign promoters in transfected cells.

The cis- element $\alpha \mathrm{BE} 1$ (-420/-396) binds GR and presumably contributes to the widespread expression of the $\alpha B$-crystallin gene (Bhat and Nagineni, 1989, Dubin et al., 1989). This sequence is contained within the $-426 /-257$ enhancing region that is responsible for $\alpha \mathrm{B}$-crystallin promoter activity in all tissues but lens and cornea during development of transgenic mice (GopalSrivastava et al., 1995, Gopal-Srivastava et al., 2000, GopalSrivastava and Piatigorsky, 1993, Haynes et al., 1996). $\alpha B E 1$ is also within the $-465 /-398$ fragment identified previously to be critical for glucocorticoid responsiveness of the $\alpha B$-crystallin gene (Scheier et al., 1996). Deletion of this part of the enhancing region virtually abolishes the ability of the $\alpha \mathrm{B}$-crystallin promoter to function in all tissues but lens and cornea in transgenic mice (Gopal-Srivastava et al., 2000). The existence of a functional GR element within the enhancing region is consistent with the presence of functional GR in rat, mouse and human lens epithelial cells (Gupta and Wagner, 2003, James et al., 2003) and with the accumulation of $\alpha \mathrm{B}$-crystallin mRNA over a period of several days in cultured non-lens cells treated with the synthetic glucocorticoid, dexamethasone (Nedellec et al., 2002, Scheier et al., 1996). It is noteworthy that the $\alpha B E 1$ element has a bidirectional positive influence on the flanking $\alpha \mathrm{B}$-crystallin and the HspB2 promoters. Whether $\alpha B E 1$, located within the orientation-dependent enhancing region (Swamynathan and Piatigorsky, 2002), is orientationdependent remains to be tested.

The significant reduction of $\alpha \mathrm{B}$-crystallin promoter activity and a modest, variable and cell type dependent elevation of HspB2 promoter activity upon mutagenesis of $\alpha B E 3$ indicate that $\alpha B E 3$ selectively activates the $\alpha \mathrm{B}$-crystallin promoter, consistent with 
A



Wild Type -738 CTGCACACCCTTCAGCTGTCCAGGGGCCTGGTAGGACAgCTGAGGGTCCTGGTC E-Box1 Mut -738 CTGCACACCCGGTGCAATCACAGGGGCCTGGTAGGACAGCTGAGGGTCCTGGTC E-Box2 Mut -738 CTGCACACCCTTCAGCTGTCCAGGGGCCTGGTAGATGTGATCCAGGTCCTGGTC E-Box1\&2 Mut -738 CTGCACACCCGGTGCAATCACAGGGGCCTGGTAGATGTGATCCAGGTCCTGGTC

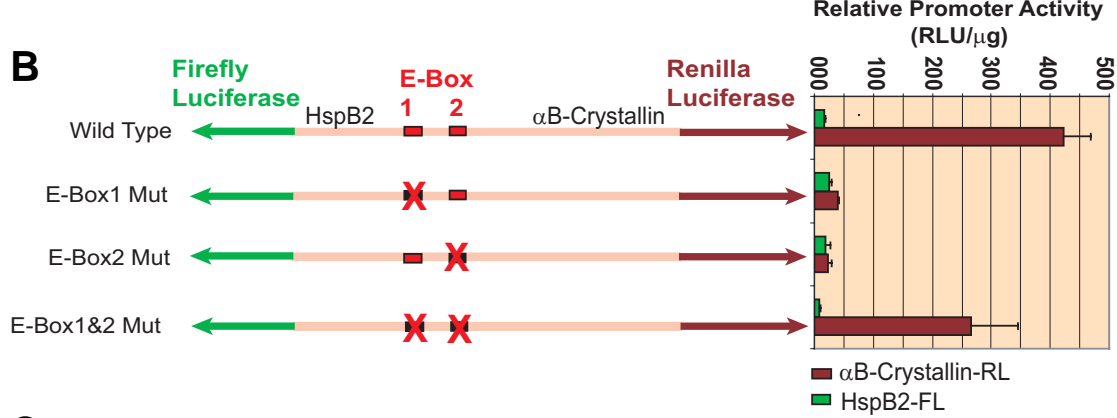

C

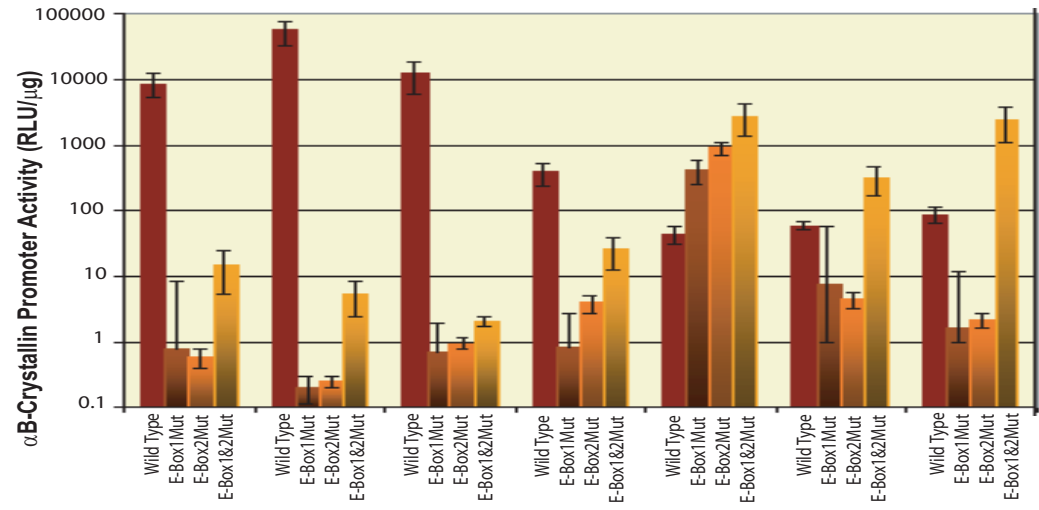

D

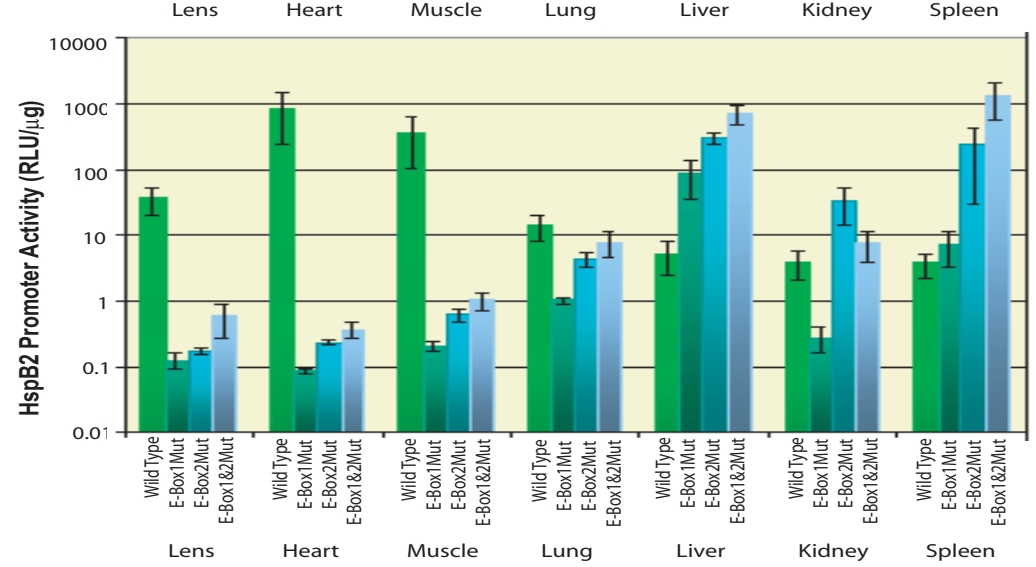

Fig. 7. Influence of the $-726 /-721$ and $-702 /-697$ E-box elements on $\alpha B$-crystallin and HspB2 promoter activities in transfected cells and transgenic mice. (A) Sequence of wild type (shown in bold) and mutated (shown in red italics) E-box elements. (B) Schematic representation of the bidirectional dual luciferase reporter vectors used is shown on the left. Relative $\alpha B$-crystallin and HspB2 promoter activities in C2C12 cells transfected with different plasmids as indicated, presented as relative luciferase units per microgram protein $(R L U / \mu g)$ in the cell extract used for the assay, is shown on the right. (C) $\alpha B$-crystallin promoter-driven Renilla luciferase reporter gene activities in the lens, heart, skeletal muscle, lung, liver, kidney and spleen of transgenic mice generated with the wild type or mutated E-box element containing intergenic region, presented as relative luciferase units per microgram protein $(R L U / \mu g)$ in the extract used. (D) HspB2 promoter-driven Firefly luciferase reporter gene activities in the lens, heart, skeletal muscle, lung, liver, kidney and spleen of transgenic mice generated with the wild type or mutated E-box element containing intergenic region, presented as relative luciferase units per microgram protein $(R L U / \mu g)$ in the extract used. Note that logarithmic scale is used for presentation of data in $(C, D)$. the preferential and orientation-dependent influence of the intergenic enhancing region on $\alpha \mathrm{B}$ crystallin promoter (Swamynathan and Piatigorsky, 2002). However, HspB2 promoter activity was activated 18-fold by PCMV-Sp1 and reduced by about 5 -fold by anti-Sp1 siRNAs in cotransfection experiments, suggesting that the HspB2 promoter is activated by Sp1. The strong positive influence of Sp1 in cotransfection assays and moderate and variable effect of mutagenesis of the Sp1 binding element $\alpha \mathrm{BE} 3$ on HspB2 promoter indicate that Sp1 may modulate the HspB2 promoter activity through additional Sp1 recognition sites present close to the HspB2 promoter (-860/-853, $-820 /-800$ and 748/-738).

Ability of E-box elements to activate (Calomme et al., 2002, Esumi et al., 2004, Kraner et al., 1998, Nguyen et al., 2003) or suppress (Garami and Gardner, 1996, Naghavi etal., 2001, Neuman et al., 1993, Tesmer et al., 1993, Yan et al., 2001, Yoshida et al., 2001) promoter activity is well documented. Indeed, our mutagenesis experiments using transgenic mice also indicate that the two E-box elements at positions -726/721 and $-702 /-697$ in the $\alpha B$-crystallin/ HspB2 intergenic region can increase or reduce promoter activity of the two genes in a tissuespecific fashion. Despite the close proximity of these E-box elements to the HspB2 promoter, their activation effect appears stronger on the distant $\alpha B$-crystallin promoter than on the HspB2 promoter due largely to the low strength of the latter relative to the former. Further studies are necessary to determine whether these E-box elements interact with myogenic regulatory factors, as might be expected considering their role in numerous muscle active E-box containing promoters (Rescan, 2001).

We suggested previously on the basis of sequence analysis and transgenic mouse results that the $-836 /-622$ intergenic sequence might insulate the HspB2 promoter from the enhancing region (Swamynathan and Piatigorsky, 2002). Our present results indicate that the $-836 /-622$ sequence acts as a negative regulatory element (NRE) rather than a classical insulator as judged by its ability to repress globin promoter activity in a position-independent fashion in transfected cells. This interpretation is consistent with the fact that the candidate CTCF binding sites in this region neither match the phylogenetic footprints among different mammals (Doerwald, 2004) nor bind CTCF (see Fig. 7 ). It thus appears that the similarity of these sequences to CTCF binding sites is fortuitous. The possibility that this putative NRE preferentially reduces the activity of its neighboring HspB2 promoter warrants further investigation. 
In addition to being a remarkable example of the numerous adaptations that have evolved to coordinate the differential expression of closely linked, divergently arranged genes, the mouse $\alpha B$-crystallin/ HspB2 intergenic region provides additional insight into the context dependency of regulatory elements. A previous example of such context dependency of cis-control elements within the $\alpha B$-crystallin/ HspB2 intergenic region was demonstrated by the orientation-dependence of the enhancing region in its natural environment (Swamynathan and Piatigorsky, 2002) and its orientation-independence when tested in a foreign environment (Dubin et al., 1991). Another example consistent with context-dependency is the striking change in tissue-specific activity of the mouse $\alpha B$-crystallin promoter that contains a two nucleotide mutation in an apparently neutral stretch at the 3 ' end of the enhancing region (Li et al., 2007). Despite that the mutated region can be deleted without eliminating lens promoter activity (Gopal-Srivastava et al., 2000), lens promoter activity is virtually abolished by the mutation when it is present in the promoter fragment (Li et al., 2007). Similarly, in the present study, sitespecific mutations within the two E-box elements in the intergenic region markedly reduce promoter strength in lens and muscle of transgenic mice, yet the activity of truncated promoters lacking these E-boxes remains intact in these tissues (Gopal-Srivastava etal., 2000, Swamynathan and Piatigorsky, 2002). It thus appears that these E-box elements may have a significant regulatory function within the natural promoter, but lens-specific activity of a truncated promoter function can compensate for their absence. It is also possible that the mutant E-box elements have simply gained a negative function by acquiring the ability to bind an inappropriate protein. The selective action of the Sp1: $\alpha \mathrm{BE} 3$ site within the enhancing region on the $\alpha \mathrm{B}$-crystallin promoter may also be an example of context dependent specialization of Sp1 regulatory function. The putative NRE within the $\alpha \mathrm{B}$-crystallinl HspB2 intergenic region represents yet another example of context dependent gene regulatory sequences. This DNA region contains multiple sites similar to the CTCF binding sites characteristic of insulators, yet we show here that it does not bind CTCF, suggesting that it is a negative regulator rather than an insulator. The absence of CTCF binding in this region supports the suggestion that DNA-binding zinc finger proteins (such as CTCF) are optimized for specificity by context dependency rather than affinity (Havranek et al., 2004). It remains to be shown whether or not the putative NRE does indeed have a negative regulatory role within its natural context in vivo.

In conclusion, the potentially striking consequences of sitespecific mutations on context- dependent utilization of gene regulatory elements have important implications for evolution. One implication is the provision of a powerful mechanism for evolving new patterns of gene expression by reassigning functional values of regulatory elements that result in novel evolutionary changes and/or new protein functions by a gene sharing mechanism (see (Piatigorsky, 2007)). The context dependency of regulatory sequences augments an already dynamic view of the genome during evolution whereby the mutation of any nucleotide may significantly change gene regulation in a quantitative and qualitative manner, even if the altered nucleotide appears to be neutral by deletion experiments or by being poorly conserved in homologous regions of other species. These far-reaching consequences of strong context-dependency of gene regulatory se- quences add considerable interest into unraveling further the interrelationships of the cis-control elements within the complex $\alpha \mathrm{B}$-crystallin/ HspB2 intergenic region.

\section{Materials and Methods}

\section{Cell culture, transfections, dual luciferase assays}

Human erythroleukemia K562 cells from Dr. Felsenfeld, NIDDK, NIH, were cultured in IMEM containing $10 \%$ fetal bovine serum (FBS), antibiotics penicillin and streptomycin and antifungal gentamycin. Murine lens epithelial $\alpha$ TN4 (Yamada et al., 1990), myoblast C2C12 (Yamada et al., 1990) and rabbit lens epithelial N/N1003 cells (Reddan et al., 1986) were maintained in Dulbeccos Modified Eagles Medium (DMEM) containing $10 \%$ FBS, antifungal gentamycin, antibiotics penicillin and streptomycin. Plasmids pCMV-Sp1 and pCMV-GR were purchased from Open Biosystems, Huntsville, AL. The sequence of siRNAs against GR and Sp1 purchased from Ambion Inc. (Austin, TX) is as follows: Sp1 siRNA (sense strand): GGUGAACUUGACCUCACAGtt, Sp1 siRNA (antisense strand): CUGUGAGGUCAAGUUCACCtg, GR siRNA (sense strand): GGUUUCUGCGUCUUCACCCtt, GR siRNA (antisense strand): GGGUGAAGACGCAGAAACCtt. Different siRNAs were transfected along with the test plasmids in co-transfection assays. All cells were grown in humidified chamber containing air with $5 \% \mathrm{CO}_{2}$ at $37^{\circ} \mathrm{C} .5 \times 10^{5}$ cells in mid-log phase of growth in $100 \mathrm{~mm}$ dishes were transfected with $1 \mu \mathrm{g}$ of plasmids using $3 \mu \mathrm{l}$ of FuGENE-6 (Roche Molecular Biochemicals, Indianapolis, IN). $10 \mathrm{ng}$ of pCMV-Gal plasmid was included in all transfections in order to normalize the efficiency of transfection across treatments. After 2 days, cells were lysed with $500 \mu$ l of passive lysis buffer (Promega, Madison, WI). Luciferase activities in $100 \mu \mathrm{g}$ (or normalized amount based on $\beta$-galactosidase assays) of lysate were measured using appropriate reagents from Promega and a Tropix TR717 microplate luminometer (Applied Biosystems, Foster City, CA). $\beta$-galactosidase assays were performed as described previously, using chlorophenol red- $\beta$-Dgalactopyranoside (CPRG) (Boehringer Mannheim, Indianapolis, IN) as the substrate (Swamynathan and Piatigorsky, 2002).

\section{Mutagenesis and construction of reporter vectors}

Unless otherwise noted, all nucleotide positions used here are relative to $\alpha B$-crystallin transcription start site. Construction and use of the bidirectional dual luciferase vector $\mathrm{pFL}-\mathrm{HSPB} 2 \alpha \mathrm{B}-\mathrm{RL}$ in which the firefly and Renilla luciferase reporter genes are under the control of intergenic $\mathrm{HspB} 2$ and $\alpha \mathrm{B}$-crystallin promoters respectively, has been described earlier (Swamynathan and Piatigorsky, 2002). Mutations in elements $\alpha B E 1, \alpha B E 3, E-b o x 1$ and E-box2 were introduced by recombinant PCR mediated ligation as described below using oligonucleotides synthesized by Integrated DNA Technologies, Coralville, IA. The intergenic fragments upstream of the elements $\alpha B E 1, \alpha B E 3, E$-box 1 or E-box 2 were amplified using:

upstream primer 8703(-959): GACTGCTGTTGCGACTAGTAGC and downstream primer:

E1 Mut-2 (5' to 3'):

\section{AGTCAAGTTGAGTGAGGACTGACTGTCCTAGAGGAGAGCAGAACTAG} E3 Mut-2:

CATGGTAGTCAGATATGAGTCTCGCATGCCAGGGGAATTGAG

E-box 1 Mut-2:

TGATTGCACCGGGTGTGCAGAGGAGGGGACTTAG, or E-box 2 Mut-2: TGGATCACATCTACCAGGCCCCTGCGACAGCTGA, respectively.

Similarly, the intergenic fragment downstream of the elements $\alpha \mathrm{BE} 1$, $\alpha B E 3$, E-box 1 or E-box 2 was amplified using:

downstream primer 8704 (+42): GGCTAGATGAATGCAGAGTC and upstream primer:

E1 Mut-1 (5' to 3'):

AGTCAGTCCTCACTCAACTTGACTGGCTAAGCCTAGGAAGATTCCAG 
E3 Mut-1:

TCATATCTGACTACCATGCACCAGCAGCTGCTTGGGATTCCA E-box 1 Mut-1: GGTGCAATCAGCAGGGGCCTGGTAGGACAGCT, or E-box 2 Mut-1: ATGTGATCCAGGTCCTGGTCCTGGAGTGAGCT, respecively.

The bold and underlined 5' half of primers E1 Mut-1, E3 Mut-1, E-box 1 Mut-1 and E-box 2 Mut-2 contain mutations in elements $\alpha B E 1, \alpha B E 3$, E-box 1 and E-box 2 and are complementary to the 5' half of primers $\mathrm{E} 1$ Mut-2, E3 Mut-2, E-box 1 Mut-2 and E-box 2 Mut-2, respectively. The upstream and downstream PCR products containing complementary mutations were then purified, mixed together, denatured, annealed and ligated by means of PCR using primers 8703 and 8704 , to generate the $-959 /+42$ bp fragment with specific mutations in element $\alpha B E 1, \alpha B E 3, E-$ box 1 or E-box 2. The fragments so produced were cloned into the Smal site of pRLFL-Null vector described previously (Swamynathan and Piatigorsky, 2002), the orientation of the intergenic fragment was tested by restriction fragment analysis and the resultant plasmids were labeled as pFL-HspB2 $\alpha \mathrm{B}-\mathrm{RL}$ E1 Mut, E3 Mut, E-box 1 Mut or E-box 2 Mut, respectively. Desired mutations were confirmed by sequencing each of the final plasmid constructs.

\section{Generation and analysis of transgenic mice}

Studies using transgenic mice were performed in accordance with the guidelines set forth by the Animal Care and Use Committee at the NEI. Plasmids pFL-HspB2 $\alpha B-R L$ E-box 1 Mut or pFL-HspB2 $\alpha B-R L$ E-box 2 Mut were digested with $\mathrm{BamHI}$ to release the fragment containing the firefly and Renilla luciferase genes under the control of the intergenic region. These fragments were eluted from agarose gels and used to generate the transgenic mice at the National Eye Institute's Transgenic Mouse Facility as described previously (Wawrousek et al., 1990). All analyses with the transgenic mice were done with approximately 8-week old mice. About $50 \mathrm{mg}$ of each tissue analyzed was homogenized in 500 $\mu \mathrm{l}$ of ice-cold $1 \mathrm{X}$ passive lysis buffer (Promega, Madison WI) on ice using a hand-held Kontes homogenizer for 30 to $45 \mathrm{sec}$. The lysate was cleared by centrifugation at $12000 \mathrm{rpm}$ for $15 \mathrm{~min}$ at $4^{\circ} \mathrm{C}$. Protein concentration in the supernatant fractions was estimated using the Bicinchoninic acid (Pierce, Rockford, IL) method. $50 \mu \mathrm{g}$ of lysate from each tissue was subjected to dual luciferase assays as above.

\section{Identification of candidate transcription factors}

Candidate transcription factors for elements $\alpha \mathrm{BE} 1$ and $\alpha \mathrm{BE} 3$ were predicted using the transcription element search software on the web available online at http://www.cbil.upenn.edu/tess and the transcription

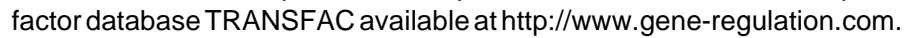

\section{Enhancer blocking assays}

Enhancer blocking assays were performed as described previously (Chung etal., 1997, Chung et al., 1993). Briefly, $1 \mu \mathrm{g}$ of linearized purified DNA was transfected using FuGENE-6 into $1 \times 10^{7}$ human erythroleukemia K562 cells grown in suspension. After 24 hours of recovery, cells were plated in $150 \mathrm{~mm}$ dishes in IMEM soft agar containing $10 \%$ FBS, antibiotics penicillin and streptomycin and geneticin at $750 \mu \mathrm{g} / \mathrm{ml}$. Colonies were counted after 3 weeks of selection and the colony number was normalized to that obtained with the $\mathrm{pNI}$ fragment containing no insert in between the enhancer and promoter.

\section{Expression and Purification of CTCF}

Full length mouse CTCF cDNA (a kind gift of Dr. Felsenfeld, NIH) was cloned in expression vector pTrcHis (Invitrogen Life Technologies, Carlsbad, CA). E. colicells containing pTrcHis:CTCF vector in mid-log phase of growth were induced to express CTCF with $1 \mathrm{mM}$ isopropyl $\beta$ D-thiogalactoside (IPTG) for 4 hours. The cells were then lysed and the expressed CTCF tagged with 6 residues of histidine was purified using the Ni-NTA purification system, following the protocol suggested by the manufacturer (Invitrogen Life Technologies, Carlsbad, CA).

\section{DNA-Protein Interactions}

Sequence of oligonucleotides used in electrophoretic mobility shift assays (EMSA) is given below with mutations shown bold and underlined.

Globin FII:

CCCAGGGATGTAATTACGTCCCTCCCCCGCTAGGGGGCAGCA

-720/-673:

CGCAGGGGCCTGGTAGGACAGCTGAGGGTCCTGGTCCTGGAGTGAGCT -720/-673 Mut:

CGCAAGTGCCTGGTAGGACAGCTGAGATTCCTGGTCCTGGAGTGAGCT -779/-725:

TATGAGGGTGTGATCAGCCTGGACCCCCTAAGTCCCCTCCTCTGCACACCCTTC -779/-725 Mut:

TATGAGICTGTGATCAGCCTGGACACTCTAAGTCCAATCCTCTGCACACTATTC -759/-723: GGACCCCCTAAGTCCCCTCCTCTGCACACCCTTCAC $-668 /-629$ :

CTCTGCAGAGGGCAAGGAGAGGACTAGTTGGGCCTTCACC

-700/-650:

GCTGAGGGTCCTGGTCCTGGAATGAGCTCCTGCTCTGCAGAGGGCAAGGAG

$-845 /-795$ :

CCACCCAAAATAGTGCAGAGCCTCTGGGGGTGGGGGAGGGCTGGGAGCCT -836/-786:

ATAGTGCAGAGCCTCTGGGGGTGGGGGAGGGCTGGGAGCCTAAGTCTAGAG aBE1: -426 TAGGACTCCACAAAGAGTTAATGTCCCTGGGGCTAA aBE3: -326 CGAGACTGGAGAGGAGGAGGGGCCCACCAG, Sp1-WT: CCCTTGGTGGGGGCGGGGCCTAAGCTGCG and Sp1-MUT: CCCTTGGTGGGTTGGGGGCCTAAGCTGCG.

Gel shift assays were performed by incubating $10 \mu \mathrm{g}$ of nuclear extract or $100 \mathrm{ng}$ of partially purified GR (Sigma Biochemicals, St. Louis, MO) or CTCF protein with $\sim 0.1 \mathrm{ng}$ double-stranded oligonucleotides end-labeled with $\gamma^{32}$ P-ATP by polynucleotide kinase in $20 \mu \mathrm{l}$ reaction mix containing $20 \mathrm{mM}$ HEPES ( $\mathrm{pH}$ 7.9), $150 \mathrm{mM} \mathrm{KCl}, 5 \mathrm{mM} \mathrm{MgCl}, 1 \mathrm{mM}$ DTT, $20 \mu \mathrm{g}$ double-stranded poly (dldC) for 30 minutes at room temperature. For super-shift assays, nuclear extracts were incubated with corresponding antibody for 2 hours at room temperature before addition of the labeled oligonucleotide and further incubation for 30 minutes at room temperature. $10 \mu \mathrm{l}$ of the reaction mix was loaded on $6 \%$ acrylamide gel in $0.5 \mathrm{X}$ TBE buffer and electrophoresed at 60 volts for 2 hours. The gels were then dried and exposed to X-ray autoradiogram.

\section{Chromatin immunoprecipitation (ChIP)}

Chromatin immunoprecipitation was performed following the EZChip protocol suggested by Upstate USA Inc., (Charlottesville, VA) using $\mathrm{C} 2 \mathrm{C} 12$ cells. DNA-bound proteins were cross-linked with DNA, chromatin was purified and sonicated to generate 200-1000-bp DNA fragments and immunoprecipitated with antibodies against Sp1, GR or IgG (Santa Cruz Biotechnology, Santa Cruz, CA, USA) using protein-A sepharose beads (Amersham Biosciences, Piscataway, NJ). The DNA-protein cross-links were reversed, DNA was purified, dissolved in TE buffer and used as a template for PCR amplification. PCR was carried out for 20 cycles using different primer pairs shown below:

-959 GACTGCTGTTGCGACTAGTAGC and

-718 CGACAGTGAAGGGTGTGCAGA,

-836 ATAGTGCAGAGCCTCTGG and

-629 ACTGGTGAAGGCCCAACT,

-739 TCTGCACACCCTTCACTGT and

-519 TTGTGGATGCTATGTGGCTCAT,

-668 CTCTGCAGAGGGCAAGGAGAGGAC and

-436 GAACTAGGTGTCTGACTG,

-546 TGTTCTATGAGCCACATAGC and

-320 GCCTGGTCTCGCATGCCAGGGGAA,

-469 ATCAGCTCAGGGTTCCAGT and

-249 ATCCTTGTTCTCTGGAGCTA, 
Intergenic elements of $\alpha B$-crystallin and $H s p B 2$ genes

\section{-299 CAGCAGCTGCTTGGGATTCCG and +44 CAGGGCTAGATGAATGCAGAGTC, -1808 ATAGAGCAGCTCGAACCCGCCA and -1528 CTCTATCACGGCTACTATGT, +3744 GAACATGGCTTCATCTCCAG and +4025 AGCTTCAGCACTAGTCACAG.}

DNA samples from sonicated nuclear lysate that underwent reverse cross-link and purification were used as input. DNA sample immunoprecipitated by antibodies against IgG was used as negative control. PCR products were separated on $1 \%$ agarose gel and visualized by staining with ethidium bromide.

\section{Acknowledgements}

We thank Drs. Stanislav Tomarev and Janine Davis, LMDB, NEI, NIH, for helpfulcomments on the manuscript and Carl Haugen and Steven Lee, $\angle M D B, N E I, N I H$ for their help with generation and maintenance of the transgenic mice. This research was supported by the Intramural Research Program, NEI, NIH.

\section{References}

ADACHI, N. and LIEBER, M.R. (2002). Bidirectional gene organization: a common architectural feature of the human genome. Cel/109: 807-9.

BELL, A.C. and FELSENFELD, G. (1999). Stopped at the border: boundaries and insulators. Curr Opin Genet Dev 9: 191-8.

BELL, A.C., WEST, A.G. and FELSENFELD, G. (1999). The protein CTCF is required for the enhancer blocking activity of vertebrate insulators. Ce//98: $387-$ 96.

BELL, A.C., WEST, A.G. and FELSENFELD, G. (2001). Insulators and boundaries: versatile regulatory elements in the eukaryotic. Science 291: 447-50.

BHAT, S.P. and NAGINENI, C.N. (1989). alpha B subunit of lens-specific protein alpha-crystallin is present in other ocular and non-ocular tissues. Biochem Biophys Res Commun 158: 319-25.

CALOMME, C., NGUYEN, T.L., DE LAUNOIT, Y., KIERMER, V., DROOGMANS, L., BURNY, A. and VAN LINT, C. (2002). Upstream stimulatory factors binding to an $E$ box motif in the $R$ region of the bovine leukemia virus long terminal repeat stimulates viral gene expression. J Biol Chem 277: 8775-89.

CHRISTENSEN, M.A., ZHOU, W., QING, H., LEHMAN, A., PHILIPSEN, S. and SONG, W. (2004). Transcriptional regulation of BACE1, the beta-amyloid precursor protein beta-secretase, by Sp1. Mol Cell Bio/24: 865-74.

CHUNG, J.H., BELL, A.C. and FELSENFELD, G. (1997). Characterization of the chicken beta-globin insulator. Proc Nat/ Acad Sci USA 94: 575-80.

CHUNG, J.H., WHITELEY, M. and FELSENFELD, G. (1993). A 5 ' element of the chicken beta-globin domain serves as an insulator in human erythroid cells and protects against position effect in Drosophila. Cel/74: 505-14.

CVEKL, A., YANG, Y., CHAUHAN, B.K. and CVEKLOVA, K. (2004). Regulation of gene expression by Pax6 in ocular cells: a case of tissue-preferred expression of crystallins in lens. Int J Dev Bio/48: 829-44.

DE JONG, W.W., CASPERS, G.J. and LEUNISSEN, J.A. (1998). Genealogy of the alpha-crystallin-small heat-shock protein superfamily. Int J Bio/ Macromo/22: 151-62.

DE JONG, W.W., HENDRIKS, W., MULDERS, J.W. and BLOEMENDAL, H. (1989). Evolution of eye lens crystallins: the stress connection. Trends Biochem Sci14: 365-8.

DOERWALD, L., VAN RHEEDE, T., DIRKS, R.P., MADSEN, O., REXWINKEL, R., VAN GENESEN, S.T., MARTENS, G.J., LUBSEN, N.H. (2004). Sequence and functional conservation of the intergenic region between the head to head genes encoding the small heat shock proteins aB-crystallin and HspB2 in the mammalian lineage. J. Mol. Evol. 59: 674-686.

DUBIN, R.A., GOPAL-SRIVASTAVA, R., WAWROUSEK, E.F. and PIATIGORSKY, J. (1991). Expression of the murine alpha B-crystallin gene in lens and skeletal muscle: identification of a muscle-preferred enhancer. Mol Cel/ Bio/11: 43409

DUBIN, R.A., WAWROUSEK, E.F. and PIATIGORSKY, J. (1989). Expression of the murine alpha B-crystallin gene is not restricted to the lens. $\mathrm{Mo} / \mathrm{Ce} / / \mathrm{Bio} / 9$ : 1083-91.

ESUMI, N., OSHIMA, Y., LI, Y., CAMPOCHIARO, P.A. and ZACK, D.J. (2004). Analysis of the VMD2 promoter and implication of E-box binding factors in its regulation. J Biol Chem 279: 19064-73.

FARRELL, C.M., WEST, A.G. and FELSENFELD, G. (2002). Conserved CTCF insulator elements flank the mouse and human beta-globin loci. $\mathrm{Mo} / \mathrm{Ce} / / \mathrm{Bio} / 22$ : 3820-31.

FILIPPOVA, G.N., FAGERLIE, S., KLENOVA, E.M., MYERS, C., DEHNER, Y. GOODWIN, G., NEIMAN, P.E., COLLINS, S.J. and LOBANENKOV, V.V. (1996). An exceptionally conserved transcriptional repressor, CTCF, employs different combinations of zinc fingers to bind diverged promoter sequences of avian and mammalian c-myc oncogenes. Mol Cell Biol16: 2802-13.

FRANCK, E., MADSEN, O., VAN RHEEDE, T., RICARD, G., HUYNEN, M.A. and DE JONG, W.W. (2004). Evolutionary diversity of vertebrate small heat shock proteins. J Mol Evo/59: 792-805.

GARAMI, M. and GARDNER, D.G. (1996). An E-box motif conveys inhibitory activity on the atrial natriuretic peptide gene. Hypertension 28: 315-9.

GOPAL-SRIVASTAVA, R., CVEKL, A. and PIATIGORSKY, J. (1996). Pax-6 and alphaB-crystallin/small heat shock protein gene regulation in the murine lens. Interaction with the lens-specific regions, LSR1 and LSR2. J Biol Chem 271: 23029-36.

GOPAL-SRIVASTAVA, R., CVEKL, A. and PIATIGORSKY, J. (1998). Involvement of retinoic acid/retinoid receptors in the regulation of murine alphaB-crystallin/ small heat shock protein gene expression in the lens. J Biol Chem 273: 1795461.

GOPAL-SRIVASTAVA, R., HAYNES, J.I., 2ND and PIATIGORSKY, J. (1995). Regulation of the murine alpha B-crystallin/small heat shock protein gene in cardiac muscle. Mol Cell Bio/15: 7081-90.

GOPAL-SRIVASTAVA, R., KAYS, W.T. and PIATIGORSKY, J. (2000). Enhancerindependent promoter activity of the mouse alphaB-crystallin/small heat shock protein gene in the lens and cornea of transgenic mice. Mech Dev92: 125-34.

GOPAL-SRIVASTAVA, R. and PIATIGORSKY, J. (1993). The murine alpha Bcrystallin/small heat shock protein enhancer: identification of alpha BE-1, alpha BE-2, alpha BE-3 and MRF control elements. Mol Cell Bio/13: 7144-52.

GOPAL-SRIVASTAVA, R. and PIATIGORSKY, J. (1994). Identification of a lensspecific regulatory region (LSR) of the murine alpha B-crystallin gene. Nucleic Acids Res 22: 1281-6.

GUPTA, V. and WAGNER, B.J. (2003). Expression of the functional glucocorticoid receptor in mouse and human lens epithelial cells. Invest Ophthalmol Vis Sci44: 2041-6.

HAVRANEK, J.J., DUARTE, C.M. and BAKER, D. (2004). A simple physical model for the prediction and design of protein-DNA interactions. JMo/ Bio/344: 59-70.

HAYNES, J.I., 2ND, DUNCAN, M.K. and PIATIGORSKY, J. (1996). Spatial and temporal activity of the alpha B-crystallin/small heat shock protein gene promoter in transgenic mice. Dev Dyn 207: 75-88.

HAYNES, J.I., 2ND, GOPAL-SRIVASTAVA, R., FREDERIKSE, P.H. and PIATIGORSKY, J. (1995). Differential use of the regulatory elements of the alpha B-crystallin enhancer in cultured murine lung (MLg), lens (alpha TN4-1) and muscle (C2C12) cells. Gene 155: 151-8.

HAYNES, J.I., 2ND, GOPAL-SRIVASTAVA, R. and PIATIGORSKY, J. (1997) alpha B-crystallin TATA sequence mutations: lens-preference for the proximal TATA box and the distal TATA-like sequence in transgenic mice. Biochem Biophys Res Commun 241: 407-13.

HORWITZ, J. (2003). Alpha-crystallin. Exp Eye Res 76: 145-53.

IWAKI, A., NAGANO, T., NAKAGAWA, M., IWAKI, T. and FUKUMAKI, Y. (1997). Identification and characterization of the gene encoding a new member of the alpha-crystallin/small hsp family, closely linked to the alphaB-crystallin gene in a head-to-head manner. Genomics 45: 386-94.

JAMES, E.R., ROBERTSON, L., EHLERT, E., FITZGERALD, P., DROIN, N. and GREEN, D.R. (2003). Presence of a transcriptionally active glucocorticoid receptor alpha in lens epithelial cells. Invest Ophthalmol Vis Sci 44: 5269-76.

KAPPE, G., FRANCK, E., VERSCHUURE, P., BOELENS, W.C., LEUNISSEN, J.A and DE JONG, W.W. (2003). The human genome encodes 10 alpha-crystallinrelated small heat shock proteins: HspB1-10. Cell Stress Chaperones 8: 53-61.

KLEMENZ, R., FROHLI, E., STEIGER, R.H., SCHAFER, R. and AOYAMA, A. 
(1991). Alpha B-crystallin is a small heat shock protein. Proc Nat/ Acad Sci USA 88: 3652-6.

KOYANAGI, K.O., HAGIWARA, M., ITOH, T., GOJOBORI, T. and IMANISHI, T. (2005). Comparative genomics of bidirectional gene pairs and its implications for the evolution of a transcriptional regulation system. Gene 353: 169-76.

KRANER, S.D., RICH, M.M., KALLEN, R.G. and BARCHI, R.L. (1998). Two Eboxes are the focal point of muscle-specific skeletal muscle type $1 \mathrm{Na}+$ channel gene expression. J Biol Chem 273: 11327-34.

LABRADOR, M. and CORCES, V.G. (2002). Setting the boundaries of chromatin domains and nuclear organization. Cel/111: 151-4.

LEE, G.R., KIM, S.T., SPILIANAKIS, C.G., FIELDS, P.E. and FLAVELL, R.A. (2006). T helper cell differentiation: regulation by cis elements and epigenetics. Immunity 24: 369-79.

LEE, G.R., SPILIANAKIS, C.G. and FLAVELL, R.A. (2005). Hypersensitive site 7 of the TH2 locus control region is essential for expressing TH2 cytokine genes and for long-range intrachromosomal interactions. Nat Immuno/6: 42-8.

LI, Y., HOUGH, R.B. and PIATIGORSKY, J. (2007). Tissue-specific activity of the blind mole rat and the two nucleotide-mutated mouse \{alpha\}B-crystallin promoter in transgenic mice. Proc Natl Acad Sci USA 104: 2608-13.

MARCO, E., GARCIA-NIETO, R. and GAGO, F. (2003). Assessment by molecular dynamics simulations of the structural determinants of DNA-binding specificity for transcription factor Sp1. J Mol Bio/328: 9-32.

NAGHAVI, M.H., ESTABLE, M.C., SCHWARTZ, S., ROEDER, R.G. and VAHLNE, A. (2001). Upstream stimulating factor affects human immunodeficiency virus type 1 (HIV-1) long terminal repeat-directed transcription in a cell-specific manner, independently of the HIV-1 subtype and the core-negative regulatory element. J Gen Viro/82: 547-59.

NEDELLEC, P., EDLING, Y., PERRET, E., FARDEAU, M. and VICART, P. (2002). Glucocorticoid treatment induces expression of small heat shock proteins in human satellite cell populations: consequences for a desmin-related myopathy involving the R120G alpha B-crystallin mutation. Neuromuscul Disord 12: 45765.

NEUMAN, T., METSIS, M., PERSSON, H. and GRUSS, P. (1993). Cell typespecific negative regulatory element in low-affinity nerve growth factor receptor gene. Brain Res Mol Brain Res 20: 199-208.

NGUYEN, Q.G., BUSKIN, J.N., HIMEDA, C.L., SHIELD, M.A. and HAUSCHKA, S.D. (2003). Differences in the function of three conserved E-boxes of the muscle creatine kinase gene in cultured myocytes and in transgenic mouse skeletal and cardiac muscle. J Biol Chem 278: 46494-505.

OHLSSON, R., RENKAWITZ, R. and LOBANENKOV, V. (2001). CTCF is a uniquely versatile transcription regulator linked to epigenetics and disease. Trends Genet 17: 520-7.

PIATIGORSKY, J. (2007). Gene Sharing and Evolution: Diversity of Protein Functions. Harvard University Press, Cambridge, MA.

RECILLAS-TARGA, F., BELL, A.C. and FELSENFELD, G. (1999). Positional enhancer-blocking activity of the chicken beta-globin insulator in transiently transfected cells. Proc Natl Acad Sci USA 96: 14354-9.

REDDAN, J.R., CHEPELINSKY, A.B., DZIEDZIC, D.C., PIATIGORSKY, J. and GOLDENBERG, E.M. (1986). Retention of lens specificity in long-term cultures of diploid rabbit lens epithelial cells. Differentiation 33: 168-74.

RESCAN, P.Y. (2001). Regulation and functions of myogenic regulatory factors in lower vertebrates. Comp Biochem Physiol B Biochem Mol Biol 130: 1-12.

SCHEIER, B., FOLETTI, A., STARK, G., AOYAMA, A., DOBBELING, U., RUSCONI, S. and KLEMENZ, R. (1996). Glucocorticoids regulate the expression of the stressprotein alpha B-crystallin. Mol Cell Endocrinol 123: 187-98.

SCHONEVELD, O.J., GAEMERS, I.C. and LAMERS, W.H. (2004). Mechanisms of glucocorticoid signalling. Biochim Biophys Acta 1680: 114-28.

SCHUG, J. (2003). Using TESS to predict transcription factor binding sites in DNA sequence. In Current Protocols in Bioinformatics, (ed. BAXEVANIS, A. D.). J.
Wiley and Sons.

SEGARD-MAUREL, I., RAJKOWSKI, K., JIBARD, N., SCHWEIZER-GROYER, G., BAULIEU, E.E. and CADEPOND, F. (1996). Glucocorticosteroid receptor dimerization investigated by analysis of receptor binding to glucocorticosteroid responsive elements using a monomer-dimer equilibrium model. Biochemistry 35: 1634-42.

SPILIANAKIS, C.G. and FLAVELL, R.A. (2004). Long-range intrachromosomal interactions in the T helper type 2 cytokine locus. Nat Immuno/5: 1017-27.

SPILIANAKIS, C.G., LALIOTI, M.D., TOWN, T., LEE, G.R. and FLAVELL, R.A. (2005). Interchromosomal associations between alternatively expressed loci. Nature 435: 637-45.

SUZUKI, A., SUGIYAMA, Y., HAYASHI, Y., NYU-I, N., YOSHIDA, M., NONAKA, I., ISHIURA, S., ARAHATA, K. and OHNO, S. (1998). MKBP, a novel member of the small heat shock protein family, binds and activates the myotonic dystrophy protein kinase. J Cell Bio/140: 1113-24.

SWAMYNATHAN, S.K. and PIATIGORSKY, J. (2002). Orientation-dependent influence of an intergenic enhancer on the promoter activity of the divergently transcribed mouse Shsp/alpha B-crystallin and Mkbp/HspB2 genes. J Biol Chem 277: 49700-6.

TAKAI, D. and JONES, P.A. (2004). Origins of bidirectional promoters: computational analyses of intergenic distance in the human genome. Mol Biol Evo/21: 463-7.

TESMER, V.M., RAJADHYAKSHA, A., BABIN, J. and BINA, M. (1993). NF-IL6mediated transcriptional activation of the long terminal repeat of the human immunodeficiency virus type 1. Proc Natl Acad Sci USA 90: 7298-302.

TRINKLEIN, N.D., ALDRED, S.F., HARTMAN, S.J., SCHROEDER, D.I., OTILLAR, R.P. and MYERS, R.M. (2004). An abundance of bidirectional promoters in the human genome. Genome Res 14: 62-6.

VOSTROV, A.A. and QUITSCHKE, W.W. (1997). The zinc finger protein CTCF binds to the APBbeta domain of the amyloid beta-protein precursor promoter. Evidence for a role in transcriptional activation. J Biol Chem 272: 33353-9.

WAWROUSEK, E.F., CHEPELINSKY, A.B., MCDERMOTT, J.B. and PIATIGORSKY, J. (1990). Regulation of the murine alpha A-crystallin promoter in transgenic mice. Dev Bio/137: 68-76.

WISTOW, G.J. and PIATIGORSKY, J. (1988). Lens crystallins: the evolution and expression of proteins for a highly specialized tissue. Annu Rev Biochem 57 : 479-504.

WRAY, G.A. (2003). Transcriptional regulation and the evolution of development. Int J Dev Bio/47: 675-84.

XI, J., FARJO, R., YOSHIDA, S., KERN, T.S., SWAROOP, A. and ANDLEY, U.P. (2003). A comprehensive analysis of the expression of crystallins in mouse retina. Mol Vis 9: 410-9.

YAMADA, T., NAKAMURA, T., WESTPHAL, H. and RUSSELL, P. (1990). Synthesis of alpha-crystallin by a cell line derived from the lens of a transgenic animal. Curr Eye Res 9: 31-7.

YAN, B., HEUS, J., LU, N., NICHOLS, R.C., RABEN, N. and PLOTZ, P.H. (2001). Transcriptional regulation of the human acid alpha-glucosidase gene. Identification of a repressor element and its transcription factors Hes-1 and YY1. J Biol Chem 276: 1789-93.

YANG, Y., CHAUHAN, B.K., CVEKLOVA, K. and CVEKL, A. (2004). Transcriptional regulation of mouse alphaB- and gammaF-crystallin genes in lens: opposite promoter-specific interactions between Pax6 and large Maf transcription factors. J Mol Bio/344: 351-68.

YOSHIDA, I., KOIDE, S., HASEGAWA, S.I., NAKAGAWARA, A., TSUJI, A. and MATSUDA, Y. (2001). Proprotein convertase PACE4 is down-regulated by the basic helix-loop-helix transcription factor hASH-1 and MASH-1. Biochem J360: 683-9. 


\section{Related, previously published Int. J. Dev. Biol. articles \\ See our Special Issue Eye Development edited by Joram Piatigorsky and Robert Grainger at: http://www.ijdb.ehu.es/web/contents.php?vol=48\&issue=8-9}

Regulation of gene expression by Pax6 in ocular cells: a case of tissue-preferred expression of crystallins in lens Ales Cvekl, Ying Yang, Bharesh K. Chauhan and Kveta Cveklova Int. J. Dev. Biol. (2004) 48: 829-844

Lens differentiation and crystallin regulation: a chick model Hasan M. Reza and Kunio Yasuda Int. J. Dev. Biol. (2004) 48: 805-817

Additional enhancer copies, with intact cdx binding sites, anteriorize Hoxa-7/lacZ expression in mouse embryos: evidence in keeping with an instructional cdx gradient

Stephen J. Gaunt, Adam Cockley and Deborah Drage

Int. J. Dev. Biol. (2004) 48: 613-622

Germ cell-specific expression of green fluorescent protein in transgenic rainbow trout under control of the rainbow trout vasa-like gene promoter.

G Yoshizaki, Y Takeuchi, S Sakatani and T Takeuchi

Int. J. Dev. Biol. (2000) 44: 323-326

Cell-cell signaling during neurogenesis: some answers and many questions.

K M Bhat

Int. J. Dev. Biol. (1998) 42: 127-139

Developmental patterns of crystallin expression during lens fiber differentiation in amphibians.

A T Mikhailov, V N Simirskii, K S Aleinikova and N A Gorgolyuk

Int. J. Dev. Biol. (1997) 41: 883-891

A 3' remote control region is a candidate to modulate Hoxb-8 expression boundaries.

I Valarché, W de Graaff and J Deschamps

Int. J. Dev. Biol. (1997) 41: 705-714

Effects of age and genetic growth rate on the crystallin composition of the chick lens.

C Patek, M Head and R Clayton

Int. J. Dev. Biol. (1994) 38: 717-724

Lens-specific activity of the chicken delta 1-crystallin enhancer in the mouse.

Y Takahashi, K Hanaoka, K Goto and H Kondoh Int. J. Dev. Biol. (1994) 38: 365-368

Localization of delta-crystallin RNA during lens morphogenesis and differentiation in the normal and talpid3 chick embryo.

M W Head, E L Triplett, D A Ede and R M Clayton Int. J. Dev. Biol. (1992) 36: 363-372

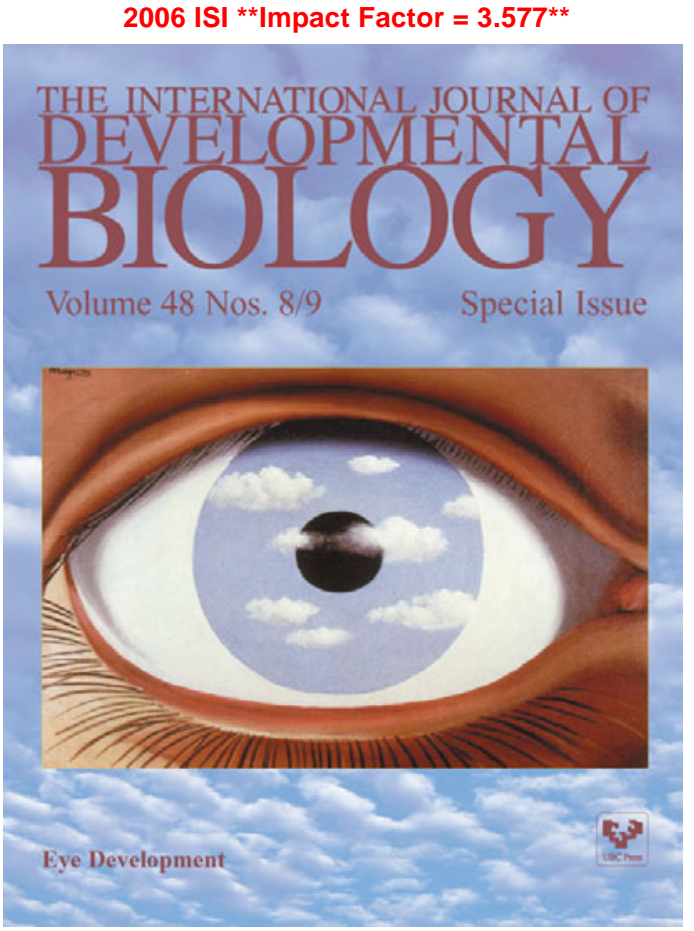

